Article

\title{
Explorative study regarding influenza vaccine hesitancy among HIV-infected patients
}

\author{
Valentina Marchese ${ }^{1, *}$, Samuele Storti ${ }^{1}$, Claudia Morganti ${ }^{1}$, Giorgio Tiecco ${ }^{1}$, Melania Degli Antoni ${ }^{1}$, Emanuele \\ Focà ${ }^{1}$, Francesco Castellì', Eugenia Quiros-Roldan ${ }^{1}$
}

1 Department of Infectious and Tropical Diseases, University of Brescia and ASST Spedali Civili of Brescia, Piazzale Spedali Civili 1, 25123 Brescia, Italy

* Correspondence: Author: v.marchese@unibs.it; valentina.marchese@ymail.com; $\underline{\text { https://orcid.org/0000-0002- }}$ $\underline{5221-1614}$

\begin{abstract}
There are scarce data regarding flu vaccination among people with HIV infection (PWHIV). The goal of this explorative study is to assess hesitancy toward influenza vaccination in a group of PWHIV during the pandemic. A questionnaire was administered to 219 patients vaccinated at our clinic during the 2020-2021 campaign. It evaluated subjects' adherence over the last 3 seasonal vaccination campaigns, vaccine confidence, complacency and convenience, and the effect of the pandemic on the choice to vaccinate. The population was divided into two groups: fully adherent (all 3 campaigns, 117 patients) and non-fully adherent (1 or 2 campaigns, 102 patients). Adherence increased in non-fully adherent group in 2020-2021, but the pandemic did not affect the choice. Misbelieves emerged: influenza vaccine was considered protective SARS-CoV-2 (22.8\% of total population); almost half of all patients thought influenza vaccine could improve their CD4+ cell level (57.3\% in fully adherent, $40.2 \%$ in non-fully adherent, $\mathrm{p}<0.05)$. A quarter of the non-fully adherent group would not have vaccinated in a location other than our clinic $(24.5 \%$ vs $11.9 \%$ in fully adherent group, $\mathrm{p}<0.05)$. Conclusively, offering a secure and private space for vaccination seems to encourage vaccination; healthcare professionals should improve counselling to increase adherence and correct misbeliefs.
\end{abstract}

Keywords: influenza vaccine; HIV; vaccine hesitancy; Italy

\section{Introduction}

Influenza virus is a common respiratory pathogen that circulates year-round and worldwide across different populations. To counteract seasonal influenza outbreaks, annual vaccination remains the primary preventive measure [1]. In Italy, influenza vaccination is recommended for subjects with a high risk of complications, such as children and adults with high-risk chronic conditions and people over 65 years old, people likely to transmit influenza to the above-mentioned subjects and workers with occupational hazards [2].

Symptoms of influenza infection appear to be similar in HIV and non-HIV patients, although the latter seems to have more frequent lower respiratory tract disease complications [3]. In patients with HIV infection (PWHIV), antiretroviral therapy (ART) decreased mortality after influenza infection by three to six-fold. Nonetheless, it remained higher than in non-HIV patients [4]. Vaccination is one of the cornerstones of public health since it is one of the most cost-effective methods to prevent infectious diseases. Although immune responses to most vaccines have been assessed to be somewhat less effective in PWHIV [5], vaccination against several infectious diseases, and among them influenza, is currently recommended for HIV infected patients [6,7]. Despite that, adherence to influenza vaccination is suboptimal and vaccination coverage hovers at a low level in nonmandatory settings [8]. 
In 2014 the World Health Organization's Strategic Advisory Group of Experts (SAGE) defined both, the concept of vaccine hesitancy (as reluctance toward vaccination despite its availability), and the factors associated (confidence, complacency and convenience)[9]. Confidence is the degree of trust in the effectiveness and safety of the vaccine, complacency, the degree to which people consider vaccination necessary to prevent a vaccine-preventable disease, and convenience the availability, affordability, willingness-to-pay, accessibility, ability to understand and accept vaccine-related information and appeal of immunization services [9].

In 2019, WHO classified vaccine hesitancy as a top 10 global health threat [10], since, determining factors of influenza vaccine hesitancy has explored in several settings [11-13]. Because vaccination is required every year due to its waning immunity and the changing strain of the virus, the evaluation of adherence and the assessment of consistency over time are crucial. In Italy, influenza vaccination for all PWHIV is free of charge after HIV diagnosis. Influenza vaccination is available in a general physician clinic or during the public immunization campaigns, but some HIV clinics (including ours) have a dedicated vaccination centre to offer all recommended vaccines for PWHIV. The simultaneous presence of the SARS-CoV-2 pandemic and the seasonal influenza epidemic caused great concern at the beginning of the 2020-2021 winter season, due to clinical similarities and possible severity in case of coinfection [14]. Unfortunately, there is scarce data about the attitude and practice of PWHIV towards influenza vaccination, especially during the COVID-19 pandemic.

This explorative study aims to investigate influenza vaccination hesitancy in a group of HIV-infected patients in a single centre to improve adherence during the next campaigns.

\section{Material and methods}

Setting and study participants

Between November and December 2020, a cross-sectional study was conducted among HIV-infected patients before the onset of the winter influenza season, via a questionnaire administered to all those PWHIV followed by our clinic (Department of Infectious and Tropical Diseases, University of Brescia, Italy) that scheduled an appointment for influenza vaccination through our outpatient offices.

COVID-19 pandemic strongly affected the Lombardia region in 2020, being Brescia one of the most affected cities, determining a delay of influenza vaccine distribution and shortage of doses compared to previous years, despite the national indication to anticipate the campaign and to extend the free of charge offer [15]. The influenza immunization 2020 campaign in Lombardia started on 19th October [16], although in our centre it was carried out from 15 November to 22 December 2020 due to dose shortage and because the second COVID-19 wave was ongoing.

\section{Data collection}

All the patients were called via telephone and were asked for verbal informed consent regarding participation in the survey, before administering the questionnaire. If a subject did not answer at first, a minimum of 3 call attempts was made to contact and evaluate as many patients as possible. During the next clinical consultation written informed consent to the survey, anonymous data collection, and publication was obtained.

A simplified questionnaire was developed based on the WHO SAGE technical report on vaccine hesitancy. The questionnaire (Suppl.1) was administered via telephone, and it included the following sections:

1.- Demographic data: gender, age;

2.- Data on Influenza vaccine adherence and attitude 
- Adherence to influenza vaccinations in the previous three seasons (2017-2018-20182019, 2019-2020), if any;

- Patient's willingness to adhere to future influenza vaccinations;

3.- Data on Influenza vaccine convenience of settings

- Location where the vaccination was carried out during the previous campaigns and patients' preferences

4.- Data on Influenza vaccine confidence, convenience other than setting, and complacency:

- Main reasons for influenza vaccine adherence or, conversely, refusal;

- Knowledge about influenza vaccine and its possible relationship with HIV infection and COVID-19.

Other items were included after that semi-structured interviews were previously conducted during clinical consultations in the vaccination clinic for PWHIV of our centre. In these interviews peculiar beliefs on the effects of vaccination on CD4+ counts, as well as confusion on influenza and COVID-19 emerged in some patients.

Since all the patients involved in this study were followed by our clinic, clinical and demographic data were retrieved from their electronic health records. The following HIVrelated data were extracted: year of the first diagnosis, last CD4+ cell count, quantitative HIV-RNA, and comorbidities, if any.

Patients were classified at first in a high adherence group (adherent to all 3 previous campaigns), a moderate adherence group (2 out of 3 campaigns) and a low adherence group, if the patient got vaccinated in only 1 out of 3 seasonal campaigns. Moreover, we classified patients as "fully adherent" (3 out of 3 campaigns) and "non-fully adherent" if they were not adherent to at least one of the former seasonal campaigns.

\section{Statistical analysis}

Absolute and relative frequency was calculated for all the categorical variables, whereas continuous variables were outlined by mean and standard deviation. All the information and data collected through the questionnaire were entered in an electronic form developed using EpiInfo (version 7). Statistical data analysis was performed using the software SPSS Statistics, version 27.0.1.0.

Patients were categorized as fully adherent ( 3 out 3 in previous campaigns) and not fully adherent (inconsistently adherence to previous campaigns) to perform univariate analysis of baseline characteristics, knowledge, and willingness responses. Chi-squared and Mann Whitney U tests were used, as appropriate $(\mathrm{p}<0.05)$.

The study obtained ethical clearance from the local Ethics Committee (study number 4722).

\section{Results}

\section{Patients' characteristics}

Out of the 3,841 HIV-infected patients censored in December 2020 as in charge at our HIV outpatient clinic, 388 booked the administration of influenza vaccine in our department for the 2020-2021 season, corresponding to $10.1 \%$ overall. All patients who have booked vaccination were contacted for the survey: $79(20.4 \%)$ were not reached after three phone call attempts and 90 (23.2\%) refused to participate to the survey, (39 patients due to time inconvenience and 51 patients consent denial). Two hundred nineteen patients $(56.4 \%)$ participated to the survey and all of them vaccinated at our clinic for the current influenza season (see Fig.1).

The mean age was 53 years (range 26-83), 76.3\% (167/219) were male, 76.5\% (58/219) had at least one comorbidity. Age was higher in the fully adherent group (mean 56.3 vs $51, \mathrm{p}<0.001)$. Cancer $(12.3 \%)$ followed by cardiovascular disease $(11 \%)$ and liver diseases $(8.7 \%)$ were the most frequent comorbidities. Years of known HIV seropositivity amounted to an average of 18 years (range 0-35 years). All subjects were on stable ART 
with plasmatic HIV viral suppression, only 3.7\% (8/219) presenting with a CD4+ cell count $\leq 200 \mathrm{cell} / \mathrm{mm}^{3}$.

\section{Influenza vaccine adherence and attitude to future vaccination}

Fourteen patients $(14 / 219,13.7 \%)$ declared they never got vaccinated during all previous three seasonal vaccine campaigns, $117 / 219$ (53.4\%) patients were "fully adherent" for the last three campaigns. Adherence was moderate (2 campaigns out of 3 ) for 25 patients $(25 / 219,11.4 \%)$ and low ( 1 campaign out of 3$)$ for 63 patients $(63 / 219,28.7 \%)$. Therefore, 102 patients $(46.6 \%)$ were not adherent to at least one of the former seasonal campaigns ("non-fully adherent").

Respect the just previous Influence vaccination campaign (2019-2020 campaign), 145 $(66.2 \%)$ patients stated adherence to previous one with a catch up of $33.8 \%$ in the current campaign from the last season. Namely, 21 out of $25(84 \%)$ of the moderate adherence group underwent vaccination in 2019-2020, while in the low adherence group only 7 out of $63(11,1 \%)$ got vaccination in the same seasonal campaign. Of the patients who had never been vaccinated before, $13.7 \%$ participated in the 2020-21 campaign, as well as $49 \%$ of those vaccinated at least once. Demographic and clinical characteristics of fully adherent and non-fully adherent patients are summarized in (see Table 1).

The attitude towards being vaccinated in the future was also evaluated. Eighty-eight per cent of interviewed patients (193/219) patients expressed the will to receive the influenza vaccine regularly over the next three years. However, patients belonging to the full adherent group showed a better attitude to vaccination compared to the counterpart $(116 / 117,99.1 \%$ vs $77 / 102,75 \%$, p=0.001).

\section{Influenza vaccine confidence and complacency (Table 2)}

Concern for a severe form of Influenza was the main declared reason for vaccination among our patients (78.5\% of patients). Fully adherent patients were more aware of the risk of developing severe forms of influenza than non-fully adherent patients (103/117, $88 \%$ vs $69 / 10262.7 \%$, p $<0.001$ ). Notwithstanding, the overall presence and the number of comorbidities did not influence the decision-making regarding influence vaccine shots (comorbidities were present in $23.1 \%$ of fully adherent vs $30.4 \%$ of non-fully adherent patients; $\mathrm{p}=0.22$ ).

A rather peculiar finding was the one concerning a common misconception among the subjects: almost half of the interviewed (49.8\%) declared that one of the reasons they were favorable to vaccination was that the vaccine could somehow raise the CD4+ cell count, thus improving their HIV-related condition $(57.3 \%$ in fully adherent group vs $40.2 \%$ in non -fully adeherent, $\mathrm{p}=0.015)$.

Regarding the current campaign, 22.7\% (50/219) patients decided to receive vaccination in the mistaken belief that the flu vaccine could also be protective against SARS-CoV2 , without statistical difference between groups. This result was confirmed in both groups (21.6\% vs $23.9 \%)$. More than half of the subjects $(112 / 219,51.1 \%)$ vaccinated for the fear of clinical overlapping with SARS CoV-2 syndrome making difficult to distinguish between them (without statistical difference between groups).

Fully adherent patients more frequently reported health-care counselling than nonadherent, reaching a borderline statistical significance $(56.4 \%$ vs $44.1 \%, \mathrm{p}=0.079)$.

Finally, only six patients $(6 / 102,5.8 \%)$ did not vaccinate due to apprehension regarding adverse events related to the vaccine or fears related to conspiracy theories tied to the anti-vaccine environment.

\section{Influenza vaccine convenience (Table 3)}

A significant reason that prompted our patients $(79 / 219,36.1 \%)$ to get vaccinated in the current campaign was the fear that healthcare facilities, in the throes of overcrowding 
due to COVID-19, would not have been able to offer an adequate standard of care in case of hospitalization (without statistical difference between the 2 groups, $36.3 \%$ vs $36 \%$ ).

A third of both the adherent and the non-adherent group declared that the need to guarantee continuity at work was a reason for vaccination and there were no meaningful differences between the two groups regarding this matter.

The availability of vaccines at our clinic was the only factor related to health care service that reached statistical significance, and almost a quarter of the non-adherent group stated that they would not have been vaccinated in a different location $(10.3 \%$ vs $25.8 \%, \mathrm{p}=0.004)$.

With regard to the previous vaccination seasons, 205 patients got the flu vaccine at least in one of the last three campaigns. More than half of these patients $(112 / 205,54.6 \%)$ received the vaccination at our healthcare facility exclusively, whilst 10/205 (4.8\%) were vaccinated in various facilities of territorial medicine: drug stores, Local Prevention department or general practitioners' clinic. The remaining part $(83,37.9 \%)$ got vaccinated by both our clinic and other facilities on different campaigns.

Sixteen out of one hundred and two subjects (16/102, 15.7\%) of the non-fully adherent group reported not having vaccinated because the vaccination shot was not available at the time of their routine HIV-infection clinical consultation.

\section{Discussion}

To our knowledge, this paper explores for the first time the hesitancy toward the influenza vaccine in PWHIV. We evaluated adherence (during the last three seasonal campaigns), knowledge and attitude expressed by the patients regarding influenza vaccine. Also, whether the pandemic has influenced their willingness to vaccinate for influence during 2020-2021 season was explored.

We observed an increase in the adherence to the influenza vaccine during the 20202021 seasonal campaign compared to previous campaigns, with a catch up from the 20192020 campaign of $33.8 \%$ of patients. Namely, $13.7 \%$ of the patients who never previously vaccinated, as well as $49 \%$ of those vaccinated at least once during the previous last three seasons, participated in the 2020-21 influenza vaccination campaign.

Several studies focused on the general population, groups at risk and general practitioners [17-21] identified hesitance as a critical obstacle to vaccination. The lack of confidence, usually due to fear of side effects and doubts about the safety and efficacy of the influence vaccine, puts the success of vaccination programs at risk $[22,23]$.

Vaccination coverage is a growing concern in Italy, with coverage against influenza in people over-65 years and chronic patients being about 55\% and $25-30 \%$ respectively in recent seasons, although an increase to $65.3 \%$ for elderly and to $23.6 \%$ for the general population has been registered in the 2020-2021 seasonal campaign [24,25].

In this survey, the setting of vaccination for PWHIV emerged as a crucial factor for vaccination adherence to preserve their own privacy. At least a quarter of the population in the non-adherent group declared they would not get vaccinated in a location other than our clinic. In Italy, Influenza vaccination is free of charge for all PWHIV independently of age or other comorbidities, but it is necessary to declare the HIV status, thus exposing them to possible social stigma. In our HIV clinic where our patients are followed by our staff periodically, there is a dedicated space for vaccination to avoid this problem. Here, we identified the setting as an important factor associated with hesitance in PWHIV. Offering vaccination in HIV clinics to better assure privacy, might prove helpful to increase adherence to all vaccinations, not just influenza.

Contrary to previous studies, the presence and number of comorbidities did not positively influence decision-making among our population [26]. Comorbidities for whom vaccination is usually recommended and the risks they entail are not correctly perceived by the patients $[27,28]$. This is confirmed by the recent literature regarding the Italian context that highlights a clear gap in knowledge and information that should be appropriately tackled [29]. 
Although the COVID-19 pandemic did not seem to be a determining factor in increasing influenza vaccine adherence, it should be noted that almost a quarter of the patients in our study were convinced that influenza vaccine was protective for SARS CoV-2 too, without any statistical difference between the two groups. Therefore, the concept of improving information and counselling should be even more stressed to allow patients to understand their disease and realize why they should vaccinate.

Indeed, only half the population that participated in the survey was brought to vaccination by the counselling offered by healthcare professionals. This endorses the fact that a proactive attitude toward vaccination by healthcare professionals and informational campaigns should be considered instrumental to boost adherence to vaccination. Furthermore, improved information and counselling could result in increased adherence by those patients who claimed not to have been vaccinated out of ignorance that the vaccine was recommended $(90 / 102,88 \%)$.

The decision-making process followed by the patients to get vaccinated is immersed in a context of beliefs and perceptions. About half of the subjects interviewed said they had been vaccinated because they were convinced that the vaccine improves the immune system, that they identify in the CD4 + cells count. In literature a transitory CD4+ cells increase is described after some vaccines (including influenza vaccine) in PWHIV, linked to the physiological vaccine response [30,31]. Adequate counselling about the meaning of this transitory response must be done.

The attitude toward vaccination is generally positive, although the presence of a social desirability bias cannot be excluded. Patients receiving influenza immunization in 2019 were more likely to accept the vaccine in 2020 and this seems to confirm the theory of "tendency to persistence" with vaccination in the prior year being of the most important predictors of vaccination [32].

Considering the findings of the survey, however, about a quarter of the non-adherent population is uncertain whether to undergo future vaccinations, thus representing the target for more incisive and precise counselling during the next campaign. In general, vulnerable groups are less hesitant toward vaccinations and their frequent access to the hospital environment may favor contact with physician information and awareness campaigns [33], which are particularly needed in the following seasonal campaigns. Indeed, the low report of influenza cases at global level in 2020-2021 may determine an increase of complacency, thus affecting adherence [34].

The most important limitation of this study is its exploratory nature with a limited number of patients, without information about PWHIV who did not book vaccination at our clinic. The survey relied on self-report, which may be unreliable and may be affected by social desirability bias (patients may have over-reported previous vaccinations or future intention to be vaccinated). Furthermore, during 2020 the number of doses of vaccine available at our HIV clinic was lower because Lombardia Region acquired fewer doses due to the pandemic, thus hindering and delaying administration. National data have not shown a reduction in the vaccination coverage [24], but inequity in dose distribution is known as a factor that can potentially influence the benefits of influenza vaccination campaign [34]. Notwithstanding these limitations, to our knowledge, this is the first study carried out in Italy that evaluates practices and attitudes toward vaccination among PWHIV.

\section{Conclusion}

Vaccine hesitancy represents a problem for global healthcare systems and the scarce awareness of diseases' severity represents an important barrier to vaccine uptake, especially in people facing chronic conditions.

Chronic diseases offer clinicians a close relationship to patients that should be exploited to foster them to get vaccinated. PWHIV should be more actively encouraged to vaccination, especially when affected by comorbidities. The organization of dedicated and flexible vaccine sessions in HIV clinics with a private and secure environment seems to 
positively affect influenza vaccine administration. Fears connected with the COVID-19 pandemic did not influence the administration of the influenza vaccine significantly. Our findings highlight the need for vaccine campaign strategies to develop culturally appropriate and effective messages and approaches tailored to the concerns. In conclusion, high influenza vaccine hesitancy in this vulnerable group is problematic, a deeper effort by healthcare professionals toward proper counselling is imperative and further research will be needed to develop appropriate messages to motivate vaccination recipients.

Conflicts of Interest. The authors declare that they have no known competing financial interests or personal relationships that could have appeared to influence the work reported in this paper.

Funding. This research received no external funding.

Institutional Review Board Statement. The study obtained ethical clearance from the Ethics Committee of Spedali Civili of Brescia, Italy (study number 4722).

Informed Consent Statement: Informed consent was obtained from all subjects involved in the study.

Availability of data and material (data transparency): the data that support the findings are available from the corresponding author, upon reasonable request.

Acknowledgments: we are grateful to all health assistances and nurses working at the HIV clinic for their contribution to the influenza seasonal campaign.

Authors' contribution: Conceptualization, VM, EQR; methodology, VM, EQR; validation, VM, EQR; formal analysis, VM; investigation, VM, EQR; data curation, CM, SS; writing - original draft preparation, VM, EQR; writing - review and editing, SS, CM, DC, EF, FC; visualization, SS; supervision, EQR; project administration, EQR. All authors have read and agreed to the published version of the manuscript.

\section{References}

1. Budd A, Blanton L, Grohskopf L, Campbell A; Dugan V; Wentworth DE; Brammer L. Centre for Disease Control, Manual for the Surveillance of Vaccine-Preventable Diseases, Chapter \& Influenza. Available at https://www.cdc.gov/vaccines/pubs/surv-manual/chpt06-influenza.html [accessed 14th September 2021]

2. Italian ministry of health. Prevenzione e controllo dell'influenza: raccomandazioni per la stagione 2020-2021 [Prevention and control of influenza: recommendation for season 2020-2021] Italian. Available at

https://www.trovanorme.salute.gov.it/norme/renderNormsanPdf?anno=2020\&codLeg=74 451\&parte=1\%20\&serie=null [accessed 14th September 2021].

3. Cohen C, Moyes J, Tempia S, Groome M, Walaza S, Pretorius M, Dawood H, Chhagan M, Haffejee S, Variava E; et al. Mortality amongst patients with influenza-associated severe acute respiratory illness, South Africa, 2009-2013. PLoS One. 2015 Mar 18;10(3):e0118884. doi: 10.1371/journal.pone.0118884. PMID: 25786103; PMCID: PMC4365037.

4. González Álvarez DA, López Cortés LF, Cordero E. Impact of HIV on the severity of influenza. Expert Rev Respir Med. 2016 Apr;10(4):463-472. doi:

10.1586/17476348.2016.1157474. Epub 2016 Mar 10. PMID: 26918376.

5. Crum-Cianflone NF, Iverson E, Defang G, Blair PJ, Eberly LE, Maguire J, Ganesan A, Faix D, Duplessis C, Lalani T, Whitman T, Brandt C, Macalino G, Millar EV, Burgess T. Durability of antibody responses after receipt of the monovalent 2009 pandemic influenza A (H1N1) vaccine among HIV-infected and HIV-uninfected adults. Vaccine. 2011 Apr 12;29(17):3183-91. doi: 10.1016/j.vaccine.2011.02.040. Epub 2011 Mar 1. PMID: 21371580; PMCID: PMC3078993.

6. Kunisaki, Ken M, and Edward N Janoff. “Influenza in Immunosuppressed

Populations: a Review of Infection Frequency, Morbidity, Mortality, and Vaccine Responses." The Lancet Infectious Diseases, vol. 9, no. 8, 2009, pp. 493-504., doi:10.1016/s1473-3099(09)70175-6.

7. Ryom L, Cotter A, De Miguel R, Béguelin C, Podlekareva D, Arribas JR, Marzolini C, Mallon P, Rauch A, Kirk O, Molina JM, Guaraldi G, Winston A, Bhagani S, Cinque P, Kowalska JD, Collins S, Battegay M; EACS Governing Board. 2019 update of the European AIDS Clinical Society Guidelines for treatment of people living with HIV version 10.0. HIV Med. 2020 Nov;21(10):617624. doi: 10.1111/hiv.12878. Epub 2020 Sep 3. PMID: 32885559; PMCID: PMC7754379.

8. Sticchi L, Bruzzone B, Caligiuri P, Rappazzo E, Lo Casto M, De Hoffer L, Gustinetti G, Viscoli C, Di Biagio A. Seroprevalence and vaccination coverage of vaccine-preventable diseases in perinatally HIV-1-infected patients. Hum Vaccin Immunother. 2015;11(1):263-9. doi: 10.4161/hv.36162. Epub 2014 Nov 1. PMID: 25483544; PMCID: PMC4514310.

9. World Health Organization. Report of the SAGE Working Group on Vaccine Hesitancy 
Available

https://www.who.int/immunization/sage/meetings/2014/october/1 Report WORKING GROUP vaccine hesitancy final.pdf [accessed 14th September 2021]

10. WHO: Anti-Vaccine Movement a Top World Threat in 2019." U.S. News \&amp; WorldReport, U.S. News \&amp; World Report, www.usnews.com/news/national-news/articles/2019-01-16/who-names-vaccine-hesitancy-astop-world-threat-in-2019.

11. Dini G, Toletone A, Sticchi L, Orsi A, Bragazzi NL, Durando P. Influenza vaccination in healthcare workers: A comprehensive critical appraisal of the literature. Hum Vaccin Immunother. 2018 Mar 4;14(3):772-789. doi: 10.1080/21645515.2017.1348442. Epub 2017 Oct 20. PMID: 28787234; PMCID: PMC5861785.

12. Domnich A, Cambiaggi M, Vasco A, Maraniello L, Ansaldi F, Baldo V, Bonanni P, Calabrò GE, Costantino C, de Waure C, Gabutti G, Restivo V, Rizzo C, Vitale F, Grassi R. Attitudes and Beliefs on Influenza Vaccination during the COVID-19 Pandemic: Results from a Representative Italian Survey. Vaccines (Basel). 2020 Nov 30;8(4):711. doi: 10.3390/vaccines8040711. PMID: 33266212; PMCID: PMC7712959.

13. Lytle KL, Collins SP, Feldstein LR, Baughman AH, Brown SM, Casey JD, Erickson HL, Exline MC, Files DC, Gibbs KW, Ginde AA, Gong MN, Grijalva CG, Khan A, Lindsell CJ, Peltan ID, Prekker ME, Rice TW, Shapiro NI, Steingrub JS, Stubblefield WB, Tenforde MW,

Womack KN, Patel MM, Self WH. Influenza and Other Viruses in the Acutely Ill (IVY) Network. Influenza vaccine acceptance and hesitancy among adults hospitalized with severe acute respiratory illnesses, United States 2019-2020. Vaccine. 2021 Aug

31;39(37):5271-5276. doi: 10.1016/j.vaccine.2021.07.057. Epub 2021 Aug 7. PMID: 34376307.

14. Dadashi M, Khaleghnejad S, Abedi Elkhichi P, Goudarzi M, Goudarzi H, Taghavi A, Vaezjalali M, Hajikhani B. COVID-19 and Influenza Co-infection: A Systematic Review and Meta-Analysis. Front Med (Lausanne). 2021 Jun 25;8:681469. doi: 10.3389/fmed.2021.681469. PMID: 34249971; PMCID: PMC8267808.

15. Italian Ministry of Health. Prevenzione e controllo dell'influenza: raccomandazioni per la stagione 2020-2021 [Prevention and control of influenza: recommendations for the season 2020-2021] Italian. Available at https://www.trovanorme.salute.gov.it/norme/renderNormsanPdf?anno=2020\&codLeg=74451\&parte=1\%20\&serie=null [accessed $14^{\text {th }}$ September 2021]

16. Regione Lombardia, Informazioni sulla campagna vaccinale. [Seasonal influenza vaccination campaign]. Italian. Available at: https://www.ats-brescia.it/campagna-vaccinale-antinfluenzale [accessed $14^{\text {th }}$ September 2021]

17. Nowak SA, Gidengil CA, Parker AM, Matthews LJ. Association among trust in healthcare providers, friends, and family, and vaccine hesitancy. Vaccine. 2021 Aug 26:S0264-410X(21)01068-9. doi: 10.1016/j.vaccine.2021.08.035. Epub ahead of print.

PMID: 34456072.

18. González-Block MÁ, Pelcastre-Villafuerte BE, Riva Knauth D, Fachel-Leal A, ComesY, Crocco P, Noboa L, Rodríguez Zea B, Ruoti M, Díaz Portillo SP, Sarti E. Influenza vaccination hesitancy in large urban centers in South America. Qualitative analysis of confidence, complacency and convenience across risk groups. PLoS One. 2021 Aug 12;16(8):e0256040. doi: 10.1371/journal.pone.0256040. PMID: 34383834; PMCID:

PMC8360613.

19. Hudson A, Montelpare WJ. Predictors of Vaccine Hesitancy: Implications for

COVID-19 Public Health Messaging. Int J Environ Res Public Health. 2021 Jul

29;18(15):8054. doi: 10.3390/ijerph18158054. PMID: 34360345; PMCID: PMC8345367.

20. Ziello A, Scavone C, Di Battista ME, Salvatore S, Di Giulio Cesare D, Moreggia O,

Allegorico L, Sagnelli A, Barbato S, Manzo V, Capuano A, Maniscalco GT. Influenza Vaccine Hesitancy in Patients with Multiple Sclerosis: A Monocentric Observational Study.

Brain Sci. 2021 Jul 5;11(7):890. doi: 10.3390/brainsci11070890. PMID: 34356125; PMCID: PMC8301852.

21. Figueroa-Parra G, Esquivel-Valerio JA, Santoyo-Fexas L, Moreno-Salinas A,Gamboa-Alonso CM, De Leon-Ibarra AL, GalarzaDelgado DA. Knowledge and attitudes about influenza vaccination in rheumatic diseases patients. Hum Vaccin Immunother. 2021 May 4;17(5):1420-1425. doi: 10.1080/21645515.2020.1816108. Epub 2020 Sep 29. PMID:

32991221; PMCID: PMC8078649.

22. Dubé E., Gagnon D., MacDonald N. E., Eskola J., Liang X., Chaudhuri M., et al.

Strategies intended to address vaccine hesitancy: Review of published reviews. Vaccine,

2015, 33(34), 4191-4203. pmid:25896385

23. Lane, S.; MacDonald, N.E.; Marti, M.; Dumolard, L. Vaccine hesitancy around theglobe: Analysis of three years of WHO/UNICEF Joint Reporting Form data-2015-2017.

Vaccine 2018, 36, 3861-3867

24. Ministero della Salute Italiano. Trend vaccinazione antinfluenzale in Italia: confronti stagioni 1999/00-2020/21. Italian [Influenza vaccine trend in Italy 1999/00-2020/21] Italian. Available at: https://www.salute.gov.it/imgs/C_17_tavole_19_3_0_file.pdf . [accessed 14 $4^{\text {th }}$ September 2021]

25. Bonanni P, Angelillo IF, Villani A, Biasci P, Scotti S, Russo R, Maio T, Vitali Rosati G, 
Barretta M, Bozzola E, Castiglia P, Chiamenti G, Conforti G, Conversano M, Ferro A, Francia F, Macrì PG, Azzari C. Maintain and increase vaccination coverage in children, adolescents, adults and elderly people: Let's avoid adding epidemics to the pandemic: Appeal from the Board of the Vaccination Calendar for Life in Italy: Maintain and increase coverage also by re-organizing vaccination services and reassuring the population. Vaccine. 2021 Feb 22;39(8):1187-1189. doi: 10.1016/j.vaccine.2020.10.024. Epub 2020 Dec 11. PMID:

33309482; PMCID: PMC7831710.

26. Gerussi V, Peghin M, Palese A, et al. Vaccine Hesitancy among Italian Patients Recovered from COVID-19 Infection towards Influenza and Sars-Cov-2 Vaccination. Vaccines (Basel). 2021;9(2):172. Published 2021 Feb 18. doi:10.3390/vaccines9020172

27. Alcusky MJ, Pawasauskas J. Adherence to Guidelines for Hepatitis B, Pneumococcal, and Influenza Vaccination in Patients With Diabetes. Clin Diabetes. 2015 Jul;33(3):116-22. doi: 10.2337/diaclin.33.3.116. PMID: 26203204; PMCID: PMC4503939.

28. Martinez-Huedo MA, Lopez-De-Andrés A, Mora-Zamorano E, Hernández-Barrera V, Jiménez-Trujillo I, Zamorano-Leon JJ, Jiménez-García R. Decreasing influenza vaccine coverage among adults with high-risk chronic diseases in Spain from 2014 to 2017. Hum Vaccin Immunother. 2020;16(1):95-99. doi: 10.1080/21645515.2019.1646577. Epub 2019 Oct 22. PMID: 31339794; PMCID: PMC7012160.

29. Napolitano F, Della Polla G, Capano MS, Augimeri M, Angelillo IF. Vaccinations and Chronic Diseases: Knowledge, Attitudes, and Self-Reported Adherence among Patients in Italy. Vaccines (Basel). 2020 Sep 25;8(4):560. doi: 10.3390/vaccines8040560. PMID: 32992864; PMCID: PMC7711873.

30. Thitilertdecha P, Khowawisetsut L, Ammaranond P, Poungpairoj P, Tantithavorn V, Onlamoon N. Impact of Vaccination on Distribution of T Cell Subsets in Antiretroviral-Treated HIV-Infected Children. Dis Markers. 2017;2017:5729639. doi: 10.1155/2017/5729639. Epub 2017 Jun 12. PMID: 28694558; PMCID: PMC5485315.

31. Pasricha N, Datta U, Chawla Y, Singh S, Arora SK, Sud A, Minz RW, Saikia B, Singh H, James I, Sehgal S. Immune responses in patients with HIV infection after vaccination with recombinant Hepatitis B virus vaccine. BMC Infect Dis. 2006 Mar 30;6:65. doi: 10.1186/1471-2334-6-65. PMID: 16571140; PMCID: PMC1525180.

32. Nowalk MP, Zimmerman RK, Shen S, Jewell IK, Raymund M. Barriers to pneumococcal and influenza vaccination in older community-dwelling adults (2000-2001). J Am Geriatr Soc. 2004 Jan;52(1):25-30. doi: 10.1111/j.1532-5415.2004.52006.x. PMID: 14687311.

33. Abramson ZH, Cohen-Naor V. Factors associated with performance of influenza immunization among the elderly. Isr Med Assoc J. 2000 Dec;2(12):902-7. PMID: 11344771.

34. Palache A, Rockman S, Taylor B, Akcay M, Billington JK, Barbosa P; IFPMA Influenza Vaccine Supply (IFPMA IVS) task force. Vaccine complacency and dose distribution inequities limit the benefits of seasonal influenza vaccination, despite a positive trend in use. Vaccine. 2021 Sep 11:S0264-410X(21)01157-9. doi: 10.1016/j.vaccine.2021.08.097. Epub ahead of print. PMID: 4521551; PMCID: PMC843350

Table 1. Demographic and clinical characteristics of fully adherent and non-fully adherent patients.

\begin{tabular}{|c|c|c|c|c|}
\hline & $\begin{array}{l}\text { Fully Adherent } \\
(n=117,53.4)\end{array}$ & $\begin{array}{l}\text { Non-fully adherent } \\
(n=102,46.6)\end{array}$ & $\begin{array}{c}\text { Total population } \\
(\mathrm{n}=219)\end{array}$ & $p$ value \\
\hline Age (mean, SD) & $56.3(10.5)$ & $51(9.6)$ & $53,8(10.4)$ & $\leq 0.001$ \\
\hline Sex (male, $n, \%)$ & $89(76.1)$ & $78(76)$ & $167(76.3 \%)$ & 0.94 \\
\hline $\mathrm{CD} 4(\mathrm{~m}, \mathrm{SD})$ & $787(391)$ & $697(345)$ & $745(372)$ & 0.15 \\
\hline $\mathrm{CD} 4<=200$ & $2 / 116(1.7)$ & $6(5.9)$ & $8(3.7 \%)$ & 0.10 \\
\hline Comorbidities (n,\%) & $27(23.1)$ & $31(30.4)$ & $58(26.5)$ & 0.22 \\
\hline Cardiovascular disease & $14(12)$ & $10(9.8)$ & $24(11)$ & 0.61 \\
\hline Diabetes/obesity & $9(7.7)$ & $9(8.8)$ & $18(8.2)$ & 0.76 \\
\hline Chronic renal disease & $6(5.1)$ & $3(2.9)$ & $9(4.1)$ & 0.42 \\
\hline CPD & $5(4.3)$ & $3(2.9)$ & $8(3.7)$ & 0.60 \\
\hline Cancer & $15(12.8)$ & $12(11.8)$ & $27(12.3)$ & 0.81 \\
\hline Liver disease & $5(4.3)$ & $14(13.7)$ & $19(8.7)$ & $\underline{0.01}$ \\
\hline $\begin{array}{c}\text { History of SARS-CoV-2 } \\
\text { infection }\end{array}$ & $4(3.4)$ & $3(2.9)$ & $7(3.2)$ & 0.84 \\
\hline Viral load $\leq 50 \mathrm{cp} / \mathrm{mL}$ & 117 & 102 & 217 & \\
\hline $\begin{array}{c}\text { Years of known seropositivity } \\
\text { (years,SD) }\end{array}$ & $19(10.2)$ & $16.7(11.4)$ & $18(10.8)$ & 0.13 \\
\hline
\end{tabular}




$$
(\mathrm{n}=110)
$$

$(\mathrm{n}=91)$

$(\mathrm{n}=201)$

SD: standard deviation; $n$ : number

Table 2. Influenza vaccine confidence and complacency.

\begin{tabular}{|c|c|c|c|c|}
\hline & $\begin{array}{l}\text { Fully Adherent } \\
(\mathrm{n}=117,53.4)\end{array}$ & $\begin{array}{l}\text { Non- fully adherent } \\
(\mathrm{n}=102,46.6)\end{array}$ & $\begin{array}{c}\text { Total } \\
(\mathrm{n}=219)\end{array}$ & p-value \\
\hline Protection from severe forms of flu $(\mathrm{n}, \%)$ & $103(88 \%)$ & $69(67.6 \%)$ & $172(78.5 \%)$ & $\leq 0.001$ \\
\hline $\begin{array}{l}\text { Induction of CD4 increase } \\
\text { vaccination-related }(n, \%)\end{array}$ & $67(57.3 \%)$ & $41(40.2 \%)$ & $108(49.3 \%)$ & $\underline{0.015}$ \\
\hline Effectiveness on COVID-19 disease $(n, \%)$ & $28(23.9)$ & $22(21.6)$ & $50(22.8)$ & 0.7 \\
\hline $\begin{array}{l}\text { Fear of the difficulty of SARS-CoV2- } \\
\text { influenza differential diagnosis (n,\%) }\end{array}$ & $55(47)$ & $57(55.9 \%)$ & $112(51.1)$ & 0.2 \\
\hline $\begin{array}{l}\text { Recommendation from healthcare } \\
\text { professionals }(\mathrm{n}, \%)\end{array}$ & $66(56.4)$ & $45(44.1)$ & $111(50.7)$ & 0.079 \\
\hline
\end{tabular}

Table 3. Influenza vaccine convenience.

\begin{tabular}{ccccc}
\hline & $\begin{array}{c}\text { Fully Adherent } \\
(\mathbf{n = 1 1 7}, \mathbf{5 3 . 4})\end{array}$ & $\begin{array}{c}\text { Non- fully adherent } \\
(\mathbf{n = 1 0 2}, \mathbf{4 6 . 6})\end{array}$ & $\begin{array}{c}\text { Total } \\
(\mathbf{n = 2 1 9 )}\end{array}$ & p-value \\
\hline $\begin{array}{c}\text { Work issues: economic or work } \\
\text { difficulties in case of illness (n,\%) }\end{array}$ & $32(27.3)$ & $30(29.4)$ & $62(28.3)$ & 0.765 \\
\hline $\begin{array}{c}\text { Fear of limited access to care due to } \\
\text { hospital overcrowding linked to the } \\
\text { pandemic (n,\%) }\end{array}$ & $42(36)$ & $37(36.3)$ & $79(36.1)$ & 1 \\
\hline $\begin{array}{c}\text { Willingness toward vaccination in } \\
\text { other healthcare facilities (n,\%) }\end{array}$ & $104(88.9)$ & $77(75.5)$ & $181(82.6)$ & $\underline{\mathbf{0 . 0 0 9}}$ \\
\hline
\end{tabular}




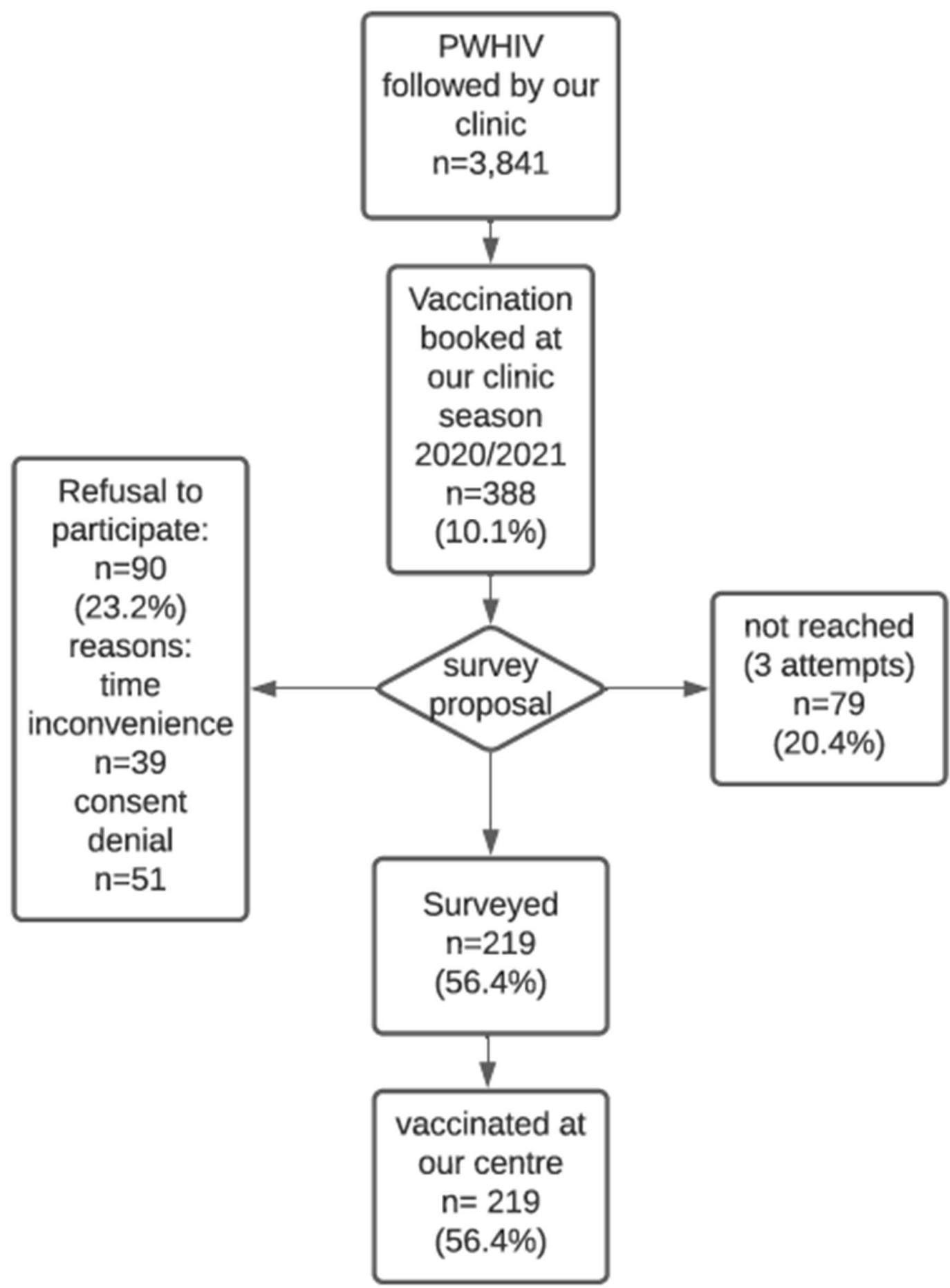

Figure 1. Flow-chart of the participation in the vaccine campaign and to the survey at the HIV clinic. 